\title{
SENSING AND INTERPRETATION TECHNIQUES
}

\author{
Marvin R. Holter \\ Institute of Science and Technology \\ The University of Michigan \\ Ann Arbor, Mich.
}

\section{Introduction}

Whatever man's ultimate goals in exploring the solar system may be, to satisfy curiosity, to locate additional habitats for the human race, or something else, achieving these objectives involves a common need. This is to map, in as much detail as possible, the morphology and constituents of the surfaces of all the planetary bodies and to determine the constituents and dynamics of the atmospheres of those bodies. For a variety of fairly obvious reasons, much of this information, at least in the foreseeable future, will have to be obtained from safe (orbital) distances, i.e., by remote sensing. Although sensors of gravitational fields, magnetic fields, and possibly other phenomena may be important, this discussion will be confined to sensors of electromagnetic radiation in the ultraviolet, visible, and infrared ranges.

Man has had centuries to map the surface and atmosphere of one body in the solar system, the earth. He has had access to practically all parts of the earth's surface and recently has achieved the ability to place sensors and human observers in orbit about the earth. In spite of all this, there is much that is not known about the earth. How then can one seriously, in a generation or two, hope to make any appreciable headway in mapping hundreds of solar system bodies? Obviously, only by effecting some striking improvements in the tools and techniques for accomplishing the task.

It is therefore disturbing to discover that improvements in the sensitivity and geometrical resolution of sensor instruments cannot provide the required increase in capability. Experience and calculation show that existing instrument techniques are adequate for even orbital use and capable of completely overloading present data interpretation capability. Furthermore, both resolution and sensitivity come close to physical limitations. Lenses and antennas operating near the Rayleigh limit are common. It is not so widely understood that presently achievable sensor sensitivities are, for the most part, also near theoretical limits. FIGURE 1 shows the sensitivities of many modern optical detectors as compared with the theoretical limit of detectivity. Many of these detectors come close to the theoretical limit of detectivity. It is well known that good modern radio frequency detectors are limited by fundamental physical processes. Therefore, not only are resolutions and sensitivities not presently limiting, but it does not appear likely that large improvements will be easily developed.

It is the thesis of this paper that improvements of the type needed can be made by more sophisticated use of existing sensitivities and resolutions 


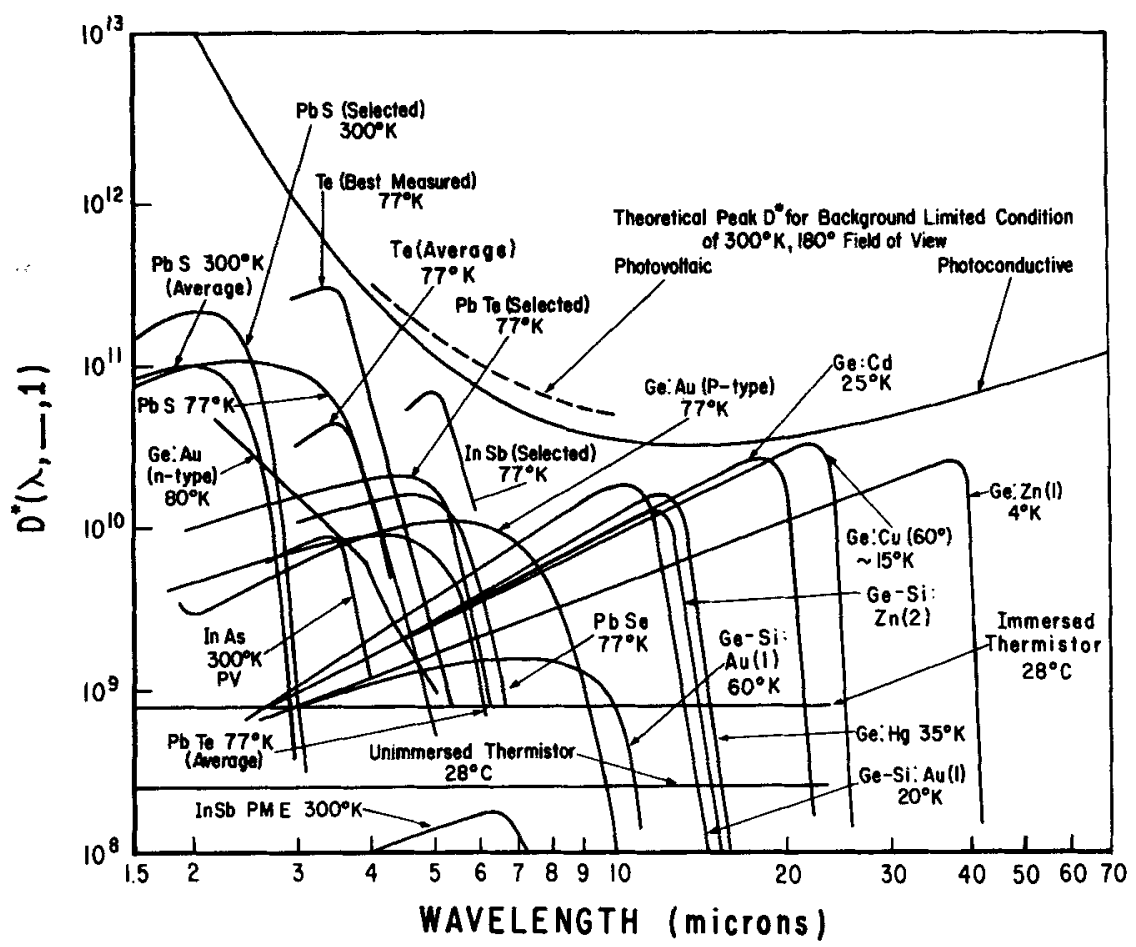

FIGURE 1 Sensitivities of Optical Detectors Compared with Theoretical Limit of Detectivity.

coupled with modern, but well developed, automatic data processing techniques. This will be demonstrated by discussing one example under development at the University of Michigan for NASA and others. The technique to be discussed is a pictorial one because the areas to be sensed are so vast. As important as they may be for special purposes, spot, nonpictorial measurements are simply not adequate to the task of mapping large areas.

\section{A Pictorial Spectrum-Matching Technique}

Pictorial sensing techniques have two major difficulties: they do not produce enough information, and simultaneously they produce too much information. They produce too little information, in that object-contrasts are neither as strong nor as numerous as they could be. This makes the task of discriminating between or identifying objects and materials more difficult than need be and in some cases not possible. They produce too much information, in that, while the average human being has an information input rate of approximately 40 bits per second, almost any sensor can produce information at megacycle rates.

By combining existing technologies in optical-mechanical scanning, spectroscopy, and automatic computation, it is possible to develop a sensing- 
interpretation system that relieves both of those difficulties simultaneously. The result is a system which, perhaps for the first time, can perform automatically a significant part of the interpretation function.

Such a combined system is sketched in FIGURE 2. The item at lower left is an optical-mechanical scanning device. The rotating flat mirror scans a small instantaneous field of view along a line in one dimension. The motion of the vehicle carrying the instrument causes successive scan lines to be displaced but contiguous, thus generating an endless television style raster scan pattern. The radiation from the scanning mirror is brought to a focus by a telescopic system. The entrance slit of a spectrometer is placed at this focus. By proper design and component selection it is possible to obtain a dispersed spectrum running from ultraviolet through the visible to and including the infrared region. Existing radiation detectors do not operate fast enough to permit superimposing a spectral scan upon the spatial scan. Therefore, the spectrum must be sampled by a number of detectors, perhaps as many as 20 or 40 , each viewing steadily a given part of the spectrum. The output of the spectrometer will then be a large number of electrical lines, each carrying a scanned picture of the same surface area but viewing the area in a different spectral band.

This configuration avoids two of the most serious problems with many multiple-sensor systems. The problem of registration of the images from the many channels does not exist, because the spectrometer entrance-slit establishes the same instantaneous field of view for all wavelengths. The usual problem of establishing spectral bandwidths by filters does not exist. The bands are established by stops in the dispersed spectrum which permit more exact tailoring of the bands than do filters.

The recording is an optional unit. In any case, it is standard technology requiring no further comment. The many electrical outputs of either the spectrometer or the recorder are routed to the amplify-and-compare unit. In addition to amplification, this unit contains well developed computertype circuitry, such as clipping and comparison circuitry, and/or gates and the like. Clearly this unit can be programmed to produce an output on a single line only when a specified combination of input signals is present. This

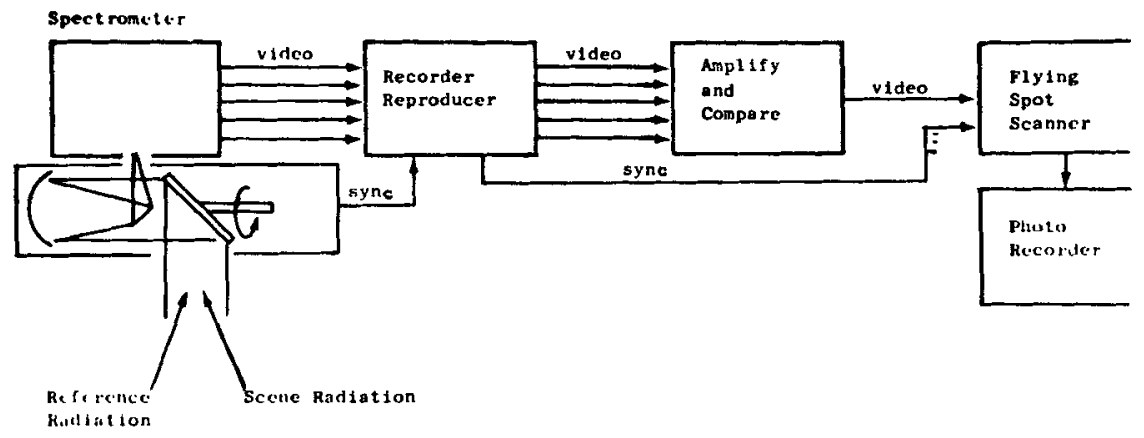

FIGURE 2 Schematic of Multispectral Scanner and Data Processor. 
specified combination might be the relative spectrum of some material of interest to the machine user. All other inputs might then be suppressed completely or presented at lowered contrast.

The output of the amplify-and-compare unit is routed to a scanning unit and photo recorder. The result is a single pictorial output whose meaningful contents have been enriched by selective use of the many spectral channels. The nature of the enrichment process is easily controlled by the human operator.

It is clear that this system embodies components representative of existing technology. The novelty lies in their mode of interconnection. Consider now how such a system can relieve the two major problems of present pictorial sensing systems.

To show that this system can produce vastly more information than any existing pictorial sensor, consider first the simplified example: a camera with black and white film is fitted with a filter passing light in three discrete wavelength bands $b_{1}, b_{2}$ and $b_{3}$, as illustrated in FIGURE 3 . Assume the respective transmissions of these bands are $t_{1}, t_{2}$, and $t_{3}$. Also assume that the powers available in these bands are $p_{1}, p_{2}$ and $p_{3}$. The tonal information in a picture taken with this camera will reside in the film density, $d$, which is expressed by

$$
\mathbf{d}=\mathbf{p}_{1} \mathbf{t}_{1}+\mathrm{p}_{2} \mathbf{t}_{2}+\mathbf{p}_{3} \mathbf{t}_{3}=\sum_{\mathrm{i}=1}^{3} \mathrm{p}_{i} \mathbf{t}_{i}
$$

The summation process, inherent in the film exposure process, will be shown to cause a loss of information that could have been preserved by adopting another process. To show this, consider the further simplifications where each product $\mathrm{p}_{i} \mathrm{t}_{i}$ can take only values of 0 or 1 . FIGURE 4 shows the possibile conditions at the outputs of the filter but before use of film, where spectral information is still preserved. There are eight possible conditions. The same

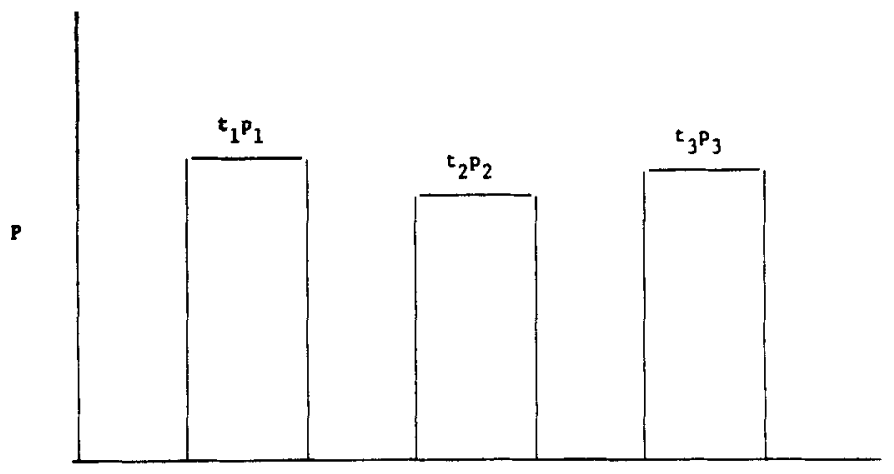

$\lambda$

FIGURE 3 Multiple Bandpass Filter Characteristics. 


$$
d=t_{1} p_{1}+t_{2} p_{2}+t_{3} p_{3}
$$

\begin{tabular}{|c|c|c|c|c|}
\hline Condition & Band 1 & Band 2 & Band 3 & $\mathrm{d}$ \\
\hline 1 & 0 & 0 & 0 & 0 \\
\hline 2 & 0 & 0 & 1 & 1 \\
\hline 3 & 0 & 1 & 0 & 1 \\
\hline 4 & 0 & 1 & 1 & 2 \\
\hline 5 & 1 & 0 & 0 & 1 \\
\hline 6 & 1 & 0 & 1 & 2 \\
\hline 7 & 1 & 1 & 0 & 2 \\
\hline 8 & 1 & 1 & 1 & 3 \\
\hline
\end{tabular}

FIGURE 4 Comparison of Information Capacities.

Figure also shows the number of possible densities after having recorded on film where spectral information is lost. There are only four recognizably different film densities because after the film integration, several of the spectrally different states are indistinguishable. Hence, in this oversimplified example, the use of film results in a loss of 50 per cent of the available information.

To extend the example to a more realistic level of complexity, assume there are $b$ possible spectral bands in which there is enough radiant power to activate a detector of some sort. These bands may lie anywhere in the ultraviolet, visible, and infrared regions. Also assume that in each band it is possible to discern $n$ levels of intensity. Then, if film is used there will be $n b$ recognizably different values of density, with any one density occurring in a number of different ways. Before recording on film there will be $n^{b}$ distinctly different possible conditions of radiant power, since differences may occur in both total level and in spectral distribution. Now $b=20$ and $n=10$ are very conservative estimates for realistic numbers of bands and discernible levels within each band. The ratio of available information before recording on film, to amount of information available on the film, is

$$
\frac{\mathrm{n}^{b}}{\mathrm{nb}}=5 \times 10^{17}
$$

In other words, such a multispectral system has an information capacity greater than conventional photography or other single-channel methods by a factor of as much as $5 \times 10^{17}$.

Permissible values for $\mathrm{n}$ and $\mathrm{b}$ were obtained by comparing detector sensitivities with available reflected sunlight and emitted infrared to determine the minimum spectral bandwidths containing sufficient radiant power to be detectable. 
This example deals only with the information capacity of an instrument and does not guarantee that nature is spectrally diverse enough to warrant an instrument of such power. Over the past several years, people at the University of Michigan and elsewhere have been observing nature in many spectral bands simultaneously. Although much work of this sort remains to be done, the indications are that natural materials are, in fact, rich enough in their spectral diversity to warrant a system of that power. FIGURES 5 and 6 are illustrative. FIGURE 5 shows field crops in several spectral bands at the U. S. Department of Agriculture experimental station adjacent to Purdue University. The changing and distinctive tone values over the several narrow visible spectral bands of some of the crops are apparent. FIGURE 6 shows Hilo, Hawaii, as viewed with panchromatic film and in the infrared in the band 4.5 to 5.5 microns. The two strips appear quite different in several respects, and these differences of tonal changes, in going from one band to the other, can form the basis for spectral signatures.

Given a system with an ability to discriminate contrasts as much as $5 \times 10^{17}$ finer than photography, and assuming that all the channels are synchronized, it takes very little familiarity with modern data processing and computer technology to realize that it is possible automatically to accomplish a great deal of materials identification, selective contrast enhancement, and other operations consituting true performance of a significant part of the

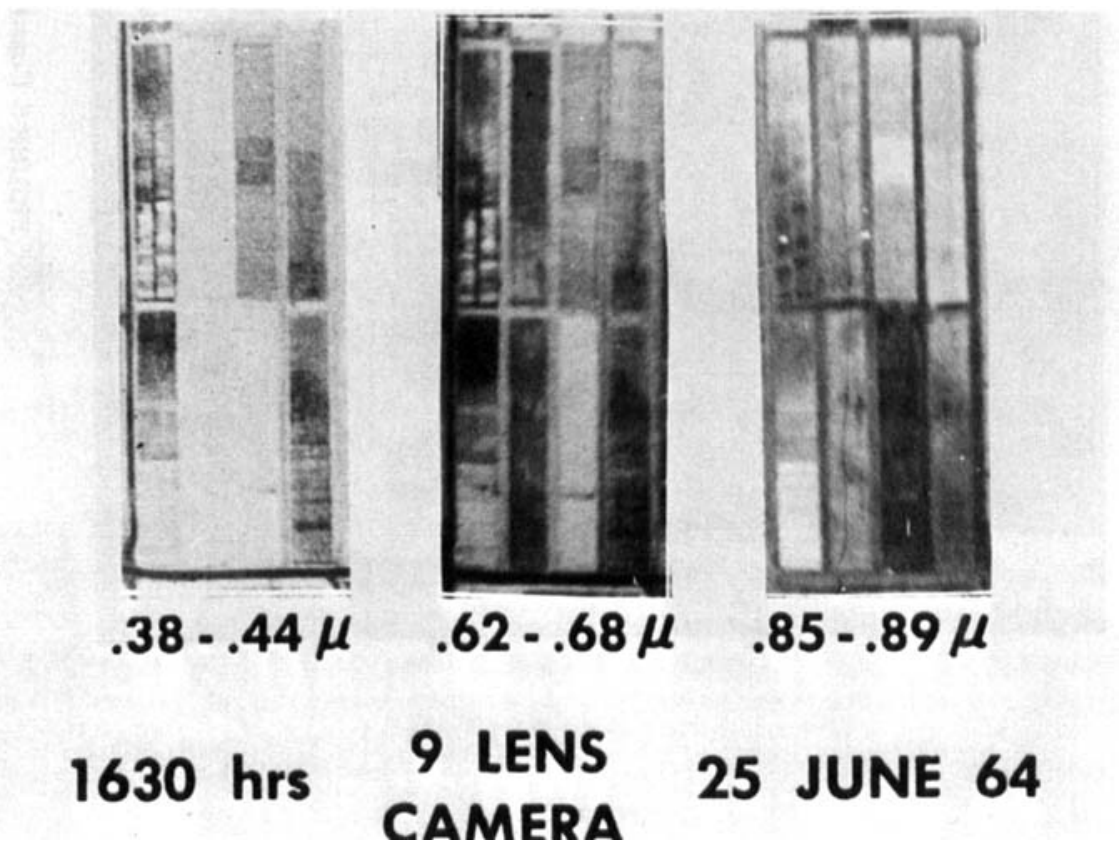

FIGURE 5 SMD of Several Crop Types. 


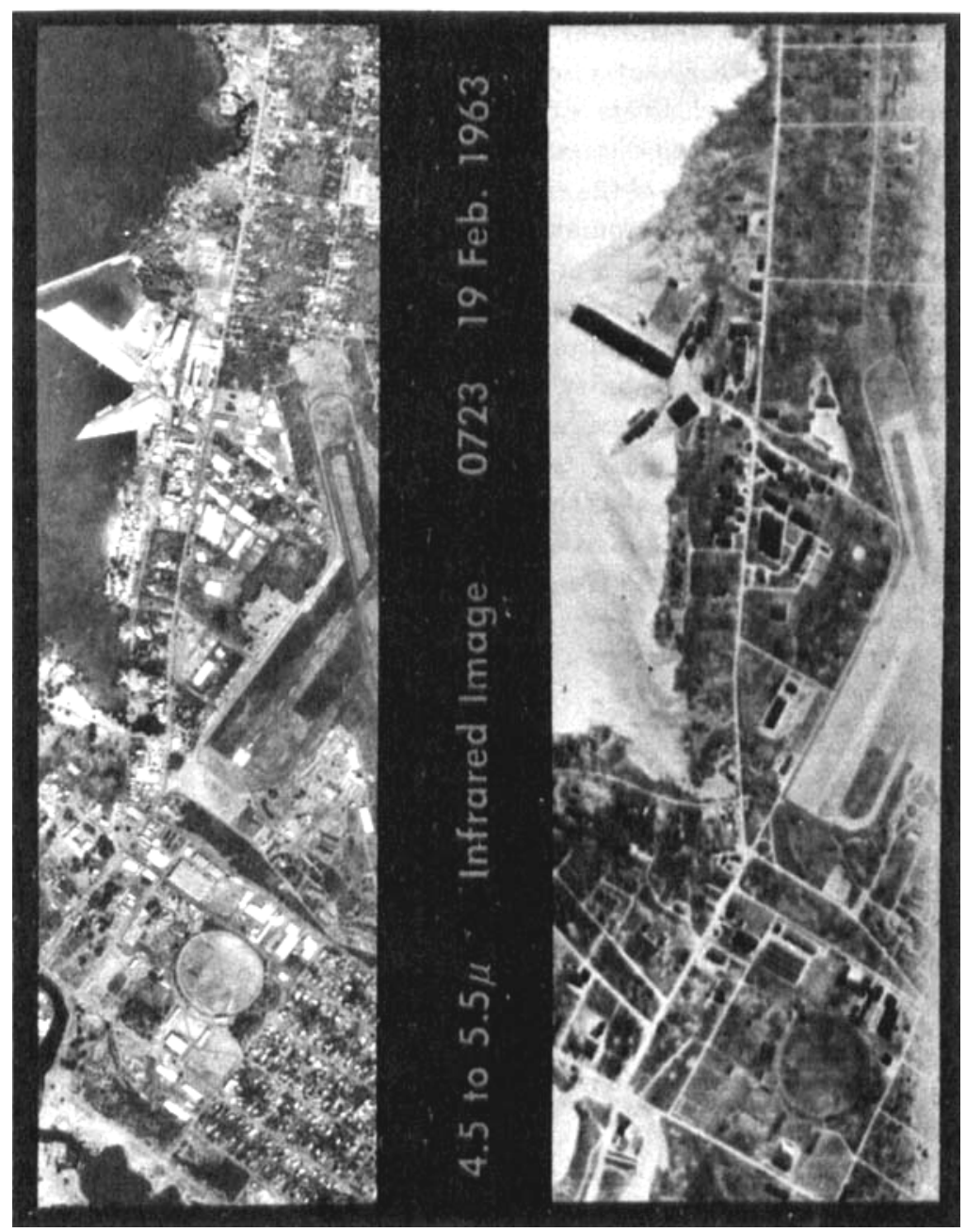

䎑 
interpretation function. This system is a true multisensor system in that, for selected purposes, it can collapse any number, $n$, of inputs into a single output. Therein lies its ability to reduce the information input requirement on the human link in the interpretation chain. Most existing so-called "multisensor" systems are, in fact, multiple-sensor systems having $\mathbf{n}$ inputs and $n$ outputs, which compound the difficulties of an initially overloaded human interpreter.

\section{Conclusion}

This discussion has attempted to show that sensing system improvements are needed, that they probably cannot be achieved by simple sensor improvements, but that the existing technology is rich enough that great improvements are possible by using it more intelligently than has been done to date. The discussion has been centered around one example: exploitation of spectral structure. Shape, time variation, polarization, and possibly other effects presumably can and should be exploited to improve and automate our ability to sense and interpret information from the large areas required.

Obviously the technique discussed creates a need for spectral signature information. In fact, the system described may be one of the best tools available for the generation, as well as employment, of spectral signature information. In any case, there exists a great need for study and understanding of spectral signatures on earth where ground verification can be carried out, so that signatures obtained where surface verification is not possible can be understood. Also the limited amount of surface measurement possible on other solar system bodies requires that those limited surface measurements be carefully planned to permit the maximum amount of extrapolation by means of orbital remote sensing. In view of the over-all task to be accomplished, it appears desirable to give first priority in surface measurements to providing "calibration" for the orbital remote sensing systems upon which reliance must be placed for gathering most of the information to be gleaned in the foreseeable future. 